\title{
A REVIEW OF TECHNIQUES IN OPTIMAL SIZING OF HYBRID RENEWABLE ENERGY SYSTEMS
}

\author{
Victor O. Okinda ${ }^{1}$, Nichodemus A. Odero ${ }^{2}$ \\ ${ }^{1}$ MSc Student, EIE Dept.UoN \\ ${ }^{2}$ Professor, EIE Dept. UoN
}

\begin{abstract}
This paper presents a review of techniques usedin recent published works on optimal sizing of hybrid renewable energy sources. Hybridization of renewable energy sources is an emergent promising trend born out of the need to fully utilize and solve problems associated with the reliability of renewable energy resources such as wind and solar. Exploitation of these resources has been instrumental in tackling or mitigating present day energy problems such as price instability for fossil based fuels, global warming and climate change in addition to being seen as way of meeting future demand for power. This paper targets researchers in the renewable energy space and the general public seeking to inform them on trends in methods applied in optimal sizing of hybrid renewable energy sources as well as to provide a scope into what has been done in this field.

In reviewing previous works, a two prong approach has been used focusing attention on the sizing methods used in the reviewed works as well as the performance indices used to check quality by these works.

In summary there is a clear indication of increased interest in recent years in optimal sizing of hybrid renewable energy resources with metaheuristic approaches such as Genetic Algorithms and Particle Swarm Optimization coming out as very interesting to researchers. It has also been observed that resources being hybridized are those with complementary regimes on specific sites.
\end{abstract}

Index Terms - Energy storage, hybrid power systems, optimization methods, renewable energy sources, reviews, solar energy, wind energy.

\section{INTRODUCTION}

The problem of optimal sizing of components of hybrid renewable energy systems is very common in recent literature. Various authors have proposed various approaches to solving this problem. Two categories for classification are presented based on the optimization method used for sizing the components of the hybrid system and on the reliability index used to ascertain reliability of the system designed.

\section{OPTIMAL SIZING ALGORITHM}

Publications in open literature using computational optimization techniques to solve renewable energy problems have increased in recent years. Various methods have been proposed, some based on more traditional approaches like mixed integer and interval linear programming, Lagrangian relaxation, quadratic programming and Nelder-Mead Simplex search. A growing number are also based on heuristic approaches especially Genetic Algorithms and Particle Swarm Optimization [1].The methods presented in the literature reviewed include:

- Dividing Rectangles (DIRECT) Search Algorithm

- Genetic Algorithms and Fuzzy Genetic Algorithms

- Particle Swarm Optimization

- Simulated Annealing

- Hybridized Solutions

- Commercial Software

- Integer Programming

\subsection{DIVIDING RECTANGLES (DIRECT) SEARCH ALGORITHM}

Dividing rectangles is a derivative-free, deterministic global optimization technique aimed at difficult global optimization problems with bound constraints and real valued objective functions. DIRECT algorithm samples points in the search space and refines the search domain on each iteration based on the samples, it thus needs no knowledge of the search space. It is a modification of the Lipschitzian optimization. A summary of works employing the DIRECT search algorithm is presented below.

Zhang et al. (2011) [2] focused on the development of a methodology for calculation of the sizing and optimization of a stand-alone hybrid system. They used DIRECT search algorithm to select from among a list of commercially available system devices, the optimal number and type of units ensuring availability of energy to meet energy demands. In their paper they used hourly data for solar radiation, wind speed and ambient temperature for a site in Le Havre, France.

Yassine et al. (2012) [3] used Dividing Rectangles (DIRECT) search algorithm to suggest from a list of commercially available systems devices, the optimal number and size of system components in a manner ensuring that the objective cost function is minimized. They considered 4 different combinations of hybrid systems.

Belfkira et al. (2007) [4] presented a new methodology for 
the design of hybrid Wind Solar PV Renewable Energy System with Battery Storage. Using DIRECT search algorithm they obtained the optimal number and type of PV cells, Wind turbines and storage units that ensured the total cost was minimized while guaranteeing the permanent availability of energy to meet demand. They modelled the configuration and implemented the direct search algorithm for optimization in Matlab®.

\subsection{GENETIC ALGORITHMS AND FUZZY GA}

Genetic Algorithms are metaheuristic search algorithms that mimic the process of evolution by natural selection. They usually start with a random generation of an initial population of chromosomes with or without domain specific knowledge. The chromosomes are represented as a data structure of binary numbers or real numbers depending on the encoding method and are parameters of possible solutions to the problem at hand. A problem specific fitness function is used to map the chromosomes into a fitness value which is a representation of the quality of the solution they represent.

Genetic Algorithm operators: Selection, Crossover and Mutation are then used to evolve the population from its current generation to the next whose average fitness value should ideally be better.

New generations are thus evolved from the knowledge of previous generations and since the fitter individuals in the population are the ones selected for crossover their good genes (good solutions) over time dominate the population and the algorithm converges to an optimum. With proper parameter selection, GA's are capable of obtaining a suitable global optimum solution.

Various variants of Genetic Algorithms exist with different subtle modifications to the original algorithm. Worth mentioning due its popularity is adaptive GA. Adaptive GA has a number of variants including fuzzy adaptive GA involves dynamic configuration of the genetic algorithm's parameters such as the mutation rate, crossover rate, or even population size depending on the status of the GA. This is in an attempt to balance and maintain precedence between exploration and exploitation. Below is a summary of literature reviewed that used Genetic Algorithms or its variants.

Yang et al. [5] had 2 main concerns whilst designing a hybrid solar-wind power generation system: the system's power reliability under varying weather conditions, and the corresponding systems cost. In their paper they proposed an optimal sizing method for the optimal configuration of a hybrid solar -wind system with battery storage using Genetic Algorithms.

Bilal et al. [6] presented the problem of optimal sizing of hybrid solar wind system with battery storage as a multiobjective optimization problem solved using Genetic Algorithms. The system was designed for an isolated site in Senegal's north coast known as Potou and its principal aims were to minimize the annualized cost of the system and to minimize the loss of power supply probability (LPSP).In their work they also investigated the influence of load profile on design, they chose three load profiles with the same daily energy. Achieved results clearly indicated that the cost of the optimal configuration was strongly dependent on the load profile.

Tafreshi et al. [7] presented a methodology to perform optimal unit sizing for Distributed Energy resources in a micro grid. They implemented a method based on Genetic Algorithms to calculate the optimal system configuration that could achieve a customer's required loss of power supply probability (LPSP) with a minimum cost of energy (COE).

Jemaa et al. (2013) [8] proposed a methodology to optimize the configuration of hybrid energy systems using fuzzy adaptive Genetic Algorithms. Fuzzy adaptive GA changes the mutation and crossover rates dynamically to ensure population diversity and prevent premature convergence. They obtained the optimal number of PV cells, wind turbines and batteries that ensures minimal total system cost whilst guaranteeing the permanent availability of energy to meet demand. They modelled the PV, wind generator and load stochastically using historical hourly wind speed, solar irradiance and load data. Their objective function to be minimized was the cost with the technical size as the constraint.

\subsection{PARTICLE SWARM OPTIMIZATION}

Particle Swarm Optimization is a population based metaheuristic optimization algorithm inspired by the social behavior of fish schooling or birds flocking. Potential solutions are represented as particles which as in the case with GA are randomly generated. Each particle has a velocity and position. Fitness function evaluations are done (the closer a particle is to the optimum the better its fitness) and each particle's best position pbest as well as the swarms best position or global best position gbest recorded. Velocities of the particles are then updated in the subsequent generation to follow the best individual. PSO and GA fall in the same category of optimization algorithms with PSO having an advantage over GA in that real valued objective functions can be used without need for encoding. Moreover proponents of PSO reckon that it is easier to implement than GA as it does not feature operator such as mutation, selection or crossover. However unlike GA, problems with more than three parameters to be optimized become more complex to represent and comprehend with PSO as it is very difficult for us to visualize or represent a particles spatiality in more than 3 dimensions. In terms of convergence and solution quality, PSO is poor in exploration (although variants of PSO that do not get stuck in local optima exists) as compared to GA but excels in exploitation of good solutions and has been shown to converge to good solutions quickly once the region containing the good solution has been narrowed down to. Below is a summary of literature reviewed that applied PSO to optimal sizing of components of hybrid renewable energy generation. 
Pirhaghshenasvali et al. [9] presented a paper in which a hybrid system for a practical standalone renewable energy generation system was proposed. They employed Wind, PV, Battery banks and Diesel Generators in their hybridization. The Wind PV Battery system was intended as the primary system with the diesel generator provided as a backup system. The goal of their optimization was to minimize investment cost and fuel cost while ensuring availability of the energy needed by the customers and sufficiency to meet peak demand. The design was based on solar radiation data, wind speed data and load curves and Particle Swarm Optimization algorithm was used for optimal sizing.

Bashir and Sadeh [10] argued that capacity sizing was important to fully meet demand due to uncertainty of wind and solar PV. They proposed a method for determining capacity of hybrid wind, PV with battery storage. Their proposed method considered uncertainty in generation of wind energy and of solar PV. They formulated the algorithm for determining the capacity of wind, PV and battery ESS as an optimization problem with the objective of minimizing the system cost whilst constrained to having a given reliability for a given load. This was solved using the PSO algorithm.

Bashir and Sadeh [11] also presented a paper in which they considered a hybrid system of wind, PV and tidal energy with battery storage. In this paper they highlight the benefits of tidal energy which is energy harnessed from rising and falling of ocean water levels as being highly predictable compared to wind and solar. They consider a 20 year plant life and optimize the design with the objective of minimizing the annualized cost of generated energy of the life of the plant, with the constraint of having a specific reliability index. They use PSO algorithm for optimization. Simulation carried out in Matlab environment revealed that in comparison to stand alone hybrid wind solar and the new system was more economical.

Saber and Venayagamoorthy [12] presented a paper suggesting the introduction of controllable loads with intelligent optimization as a necessity for the implementation of smart micro grids (SMG). They noted that over or under estimation of resources when considering reliability of the smart micro grid would make it not feasible, thus the optimization problem for sizing of SMG components was presented as a complex multi-objective optimization problem considering minimization of capital cost and operations costs as objectives subject to constraints such as net zero emission, historical wind speed and solar irradiation data and load profiles over a long period of time. They used the PSO algorithm to solve the optimization problem, and an intelligent energy management system for dispatch of the resources.

Navaerfad et al. [13] presented an optimal sizing approach for Distributed energy resources in a micro grid consisting of Wind, solar hybrid system with electrolizer, hydrogen tank, fuel cell and batteries. They proposed the uncertainty of wind power alongside a reliability index as constraints and used PSO algorithm to obtain the global optimal solution.

He, Huang and Deng [14] postulated that low carbon power technologies such as Solar - PV, Wind etc. are gaining a lot of interest and concern worldwide, and because these technologies are mainly adopted in micro grids, they emphasizes on the importance of considering the low carbon factor in the generation planning of micro grids. In this paper they use the levelized cost of electricity (LCOE) analysis method to compare the variation trends of power supplies which use different energy sources to generate in the future, and to build the energy price equilibrium point analysis model (EPE) of high carbon energy and low carbon energy. Based on the above research, the authors develops a full life cycle dynamic model for optimal sizing of components in an integrated power generation model. Modified particle Swarm optimization was used to perform optimization. Based on the three cases considered, low carbon, high carbon and equilibrium, results show that a balanced system is most suitable for clean energy production with an equilibrium point of energy consumption and carbon consumption.

\subsection{SIMULATED ANNEALING (SA)}

Simulated Annealing (SA) is a non-deterministic global optimization strategy that is inspired by the process of annealing in metallurgy. Annealing involves the heating of metals to high temperatures then followed by systematic controlled cooling to encourage formation of larger crystals with fewer defects. SA is a good strategy for finding approximate optimal solutions where a large discrete search space is involved. The procedure resembles a modified hill climber with the ability of escaping from local optima. A temperature variable is usually set high on initialization and whilst the temperature is still high the algorithm is able to accept neighboring solutions that are worse than the current solution. This allows it to explore the search space. As the temperature variable lowers, the algorithm is less likely to accept solutions that are worse than the present solution. This enables it to do exploitation of the search space in pursuit of a better solution. An acceptance function is used to determine which solution are accepted or rejected. Literature using simulated annealing method for optimization are summarized below.

Ekren and Ekren [15] used simulated annealing method for size optimization of a hybrid PV / Wind energy conversion system. Simulated annealing is a heuristic approach that uses stochastic gradient search approach for optimization. The objective function to be minimized was the hybrid system's total energy cost, with the key parameters being the PV area, wind turbine rotor sweep area and battery capacity. Results from simulated annealing were compared with those of RSM for an earlier study [16] and it was shown that simulated annealing obtained better results.

\subsection{INTEGER AND LINEAR PROGRAMMING}

Linear programming is a mathematical optimization method 
which deals with minimization or maximization of linear functions subject to linear constraints. When the decision variables of a linear program are restricted to be integers it is said to be a pure integer programing problem. When not all decision variables are restricted to be integers then it is referred to as a mixed integer linear program. In its simplest terms, a linear programming problem is solved by graphing the constraints to come up with a region known as the feasibility region within which acceptable solutions can be found. The optimization equation developed for the problem is then used to test for the most optimal point within the feasibility region. A summary of works employing variants of the linear programming method to solve for the optimal sizing of components of a hybrid renewable energy system is presented below.

Chen and Gooi, [17] proposed a new method for optimal sizing of an energy storage system. The ESS was to be used for storage of energy at times of surplus and for re-dispatch later when needed. They considered the Unit commitment problem with spinning reserve for micro grids. Their total cost function took account of the cost of the Energy Storage System (ESS), the cost of output power and the cost of spinning reserve. They formulated the main method as a mixed nonlinear integer problem (MNIP) which was solved in AMPL (A mathematical programming language). Effectiveness of the proposed method was then validated by a case study where optimal ESS rating for a micro grid were determined. Results indicated that a properly sized ESS not only stored and re-dispatched renewable energy appropriately but also reduced the total cost of the micro grid.

Bahramirad and Reder's [18] view on Energy Storage systems was that they are fast response devices that add flexibility to the control of micro grids and provide security and economic benefits to the micro grid. They thus have a major role in the long term and short term operation of micro grids. In the paper, they evaluated the benefits of ESS in islanded operation of micro grids. Long term unit commitment was then used to obtain the optimal unit scheduling. A practical model for an Energy Storage System was used and probabilistic reliability calculation method used to find expected energy not served and accordingly calculate cost of reliability of the micro grid. They solved the optimization problem using mixed integer programming method.

Chen, Gooi and Wang [19] presented a cost benefit analysis based method for optimal sizing of an energy storage system in a micro grid. They considered the unit commitment problem with spinning reserve for micro grids. Wind speed was modelled using a time series whereas solar irradiance is modelled via feed forward neural network techniques with forecasting errors being accounted for. They also presented two mathematical models for islanded and grid operation. The main problem was formulated as a mixed linear integer problem (MLIP) which is solved in AMPL. Effectiveness of the proposed method was then validated via a case study. Quantitative results indicated that optimal size for a BESS existed but differed for both grid connected and islanded mode of operation.

\subsection{RESPONSE SURFACE METHODOLOGY (RSM)}

Response surface methodology is a collection of statistical and mathematical techniques useful for the modelling and analysis of problems in which an adequate functional relationship between a response of interest and several independent input variables that influence it are developed. The objective of RSM is usually to optimize this response. There are a few attempts to using RSM to solve the problem of optimal sizing for components of a hybrid renewable energy system, these are listed below.

Ekren and Ekren (2008) [16] presented a paper aimed at showing the use of response surface methodology (RSM) in size optimization of an autonomous Wind / PV system with battery storage. The response surface output performance measure was the hybrid system cost whilst the design parameters were PV size, wind turbine rotor sweep area and battery capacity. A commercial simulation software (ARENA 10.0) was used to simulate the case study of a GSM base station. The obtained optimal results obtained by RSM were then confirmed using loss of load probability (LLP) and autonomy analysis. In a later paper [15], they compared these results with those obtained using simulated annealing method for optimization.

Ekren, Ekren and Baris [20] presented a paper to show an optimum sizing procedure for an autonomous PV/Wind hybrid energy system with battery storage. They presented a break even analysis of the system including extension of a transmission line using the net present value (NPV) method. They present a case study of a hybrid system powering a mobile communication base station. The Hybrid PV Wind system was first optimized using a response surface methodology (RSM), which is a collection of statistical and mathematical methods relying on optimization of response surface with design parameters. Optimum PV area, wind turbine rotor sweep area and battery capacity were optimally obtained using RSM, from which the total system cost was obtained. A break even analysis was then carried out to determine the distance at which transmission line extension was less economical compared to development of the hybrid power supply system. Their results showed that if the distance from the transmission line to the point of use was more than $4817 \mathrm{~m}$ then the hybrid system was more economical than the electricity network.

\subsection{HYBRIDIZED ALGORITHMS}

In some papers, methods are proposed that take advantage of complementary characteristics of two or more optimization algorithms supposedly to achieve improvements in efficiency or quality of solutions obtained. A classic example is combining GA's strength in exploration with PSO's strength in exploitation to form a hybrid GA-PSO algorithm. An overview of research works 
using hybridized algorithms is presented below.

Crăciunescu et al. [21] reckoned using Wind and Solar PV technology with battery ESS in optimally sized combination can help overcome the problem of intermittency that each of these technologies face. In the review of literature they presented, it was shown that GA and PSO were the most popular heuristic methods for optimal sizing of a hybrid wind solar power system with battery storage. In this paper, mathematical models of hybrid wind and solar were developed and multiobjective optimization performed using the intelligent search methods GA and PSO.

Arabali et al. [22] proposed a stochastic framework for optimal sizing and reliability analysis of a hybrid power system including renewable energy sources and energy storage. Uncertainties of wind and PV power generations were modeled stochastically using Auto Regressive Moving Average. Pattern Search (PS) optimization method in which scaling parameters were first initialized using GA was used in combination with sequential Monte Carlo Simulation (SMCS) to minimize system cost and satisfy system reliability requirements. SMCS simulates chronological behavior of system and emulates the reliability indices from a series of simulated experiments. They then proposed Load shifting strategies to provide some flexibility and reduce mismatch between Renewable Energy generation and Heating Ventilation and Cooling loads in the hybrid power systems. A compromise solution method was then used to arrive at the best compromise between reliability and cost. Concurrently they also ran a hybridized GA-SMCS optimizer to compare findings. The PS-SMCS optimizer outperformed GA_SMCS optimizer primarily due to PS better performance in local searches.

\subsection{COMMERCIAL SOFTWARE}

Roy et al. [23] estimated the optimal sizing for a hybrid solar - wind system for distributed generation for utilization of resources available at Sagar, a remote off-grid Island. They optimized the feasibility and size of the generation units and evaluated them using Hybrid Optimization of Multiple Energy Resources (HOMER) software. Sensitivity analysis was performed on the optimal configuration obtained. A comparison between the different modes of the hybrid system was also studied. It was estimated that the solar PV -Wind Hybrid system provided lesser cost per unit of electricity. The capital investment cost was also observed to be less when the system ran with wind DG compared to solar PV DG.

El Badawe et al. [24] aimed to optimize and model a hybrid wind solar diesel generator power system for use on remote microwave repeaters. They noted that microwave repeaters were one the main energy consumers in the telecommunication industry were usually powered by diesel generators and when these were located on remote sites, then maintenance and operations costs were even higher due to the added cost of transportation. They used HOMER software for sizing and performed sensitivity analysis to obtain the most feasible configuration, which was then modelled in SIMULINK and results presented to demonstrate system performance.

\subsection{OTHER TECHNIQUES}

Hearn et al. [25] Presented a method for sizing of grid level fly wheel energy storage using optimal control law. The method allows the loss dynamics of the flywheel to be incorporated into the sizing procedure. This permits data driven trade studies which trade peak grid power requirements and flywheel storage capacity to be performed. A case study based on home consumption and solar generation data from the largest smart grid in Austin Texas was presented.

Anagnostopoulos and Papantonis [26] advocated the attractiveness of hybrid wind hydro generation, in order to increase wind energy penetration and cost effectiveness in autonomous electric grid. In this work they used a numerical method for the optimum sizing of the various components of a reversible hydraulic system designed to recover excess electrical energy produced by wind farms and not absorbed due to grid limitations. Time variation for rejected wind farm power from a number of wind farms in Crete was used as the case study, while the free parameters considered for optimization were turbine size, size and number of pumps, penstock diameter and thickness and reservoir capacity. The proposed numerical procedure consisted of an evaluation algorithm to simulate plant operation for a 12 month period and automated optimization software based on Evolutionary Algorithms. Economic evaluation, constrained optimization, sensitivity analysis and various parametric tests were carried out on the case study. The conclusion drawn was that a well optimized design was be crucial for technical and economic viability of the system.

\section{PERFORMANCE INDEX}

Power system reliability is the system's ability to satisfy its load requirements with a reasonable assurance of continuity and quality. It is a broad field subdivided into system adequacy and system security. System security relates to the ability of the system to withstand credible contingencies without violating the normal operating limits and is generally in the context of the system's reaction to perturbations. System adequacy on the other hand is the existence of sufficient capacity within the system to meet demand [27].

In most studies to optimize the sizing components of a hybrid renewable energy power system, Generation adequacy is measured using a reliability index which quantifies the system reliability. These indices are helpful in assessing reliability performance of a generation system against some predetermined minimum requirements or reliability standards, comparing alternative designs, identifying weak spots and determining ways of improvement and in cost / performance considerations for decision making [28]. 
In the literature surveyed, two types of indices emerge, first category are those used to quantify system reliability such as the loss of load probability or expected energy not served and the other category is of those used to determine economic viability of the design such as the annualized life cycle cost and the levelized cost of electricity. A summary of these indices and the context in which they are encountered is presented below.

\subsection{LOSS OF LOAD INDICES}

According to [29], LPSP is the probability that insufficient power supply results when the hybrid system is not able to satisfy the load demand. The concept of LPSP can be summarized as, total power generated is determined (for the case of hybrid wind and PV generators)

$$
P_{t o t}(t)=P_{P V}(t)+P_{W G}(t)
$$

Inverter input power is determined as per efficiency as

$$
P_{\text {inv }}(t)=\frac{P_{\text {load }}(t)}{\eta_{\text {inv }}}
$$

Where $\eta_{\text {inv }}$ is the inverter efficiency and $P_{\text {load }}(t)$ load demand at timet.

Three cases are foreseen: Case 1 where $P_{t o t}>P_{i n v}$ and the surplus is stored in the batteries or energy storage system applied. The new storage capacity is then calculated. Case 2 is whereby $P_{t o t}<P_{i n v}$ the energy deficit is supplied by the batteries and new battery capacities calculated as they discharge for as long as the battery capacity does not decrease below a set minimum level in which case the load is shed. Finally in case 3 , in a situation where $P_{t o t}=P_{i n v}$ then storage capacity remains unchanged.

The loss of power supply at a given time interval in case 2 is calculated as

$$
\begin{aligned}
\operatorname{LPS}(t) & =P_{\text {load }}(t) \cdot \Delta t \\
& -\left(\left(P_{P V}(t)\right.\right. \\
& \left.+P_{W G}(t)\right) \Delta t \\
& +C_{\text {bat }}(t-1) \\
& \left.-C_{\text {batmin }}\right) \cdot \eta_{\text {inv }}
\end{aligned}
$$

The loss of power supply probability is evaluated as the ratio of all the LPS ( $t$ ) values over the total load required during that period. Mathematically,

$$
\operatorname{LPSP}=\frac{\sum_{t=1}^{T} \operatorname{LPS}(t)}{\sum_{t=1}^{T} P_{\text {load }(t)} \cdot \Delta t}
$$

LPSP is a very popular technical index of reliability for sizing hybrid renewable energy systems. A review of works implementing LPSP is provided below.

Yang et al. [5] used the Loss of Power Supply probability as a reliability criteria. In designing a hybrid power system to supply a remote telecommunications relay station their objective was to achieve a required loss of power supply probability (LPSP) at a minimum annualized cost of the System (ACS).
Kamjoo et al. [27] recognized the uncertainty in maintaining a high quality of supply as the main challenge in standalone hybrid renewable energy systems. They considered the Loss of Power Supply Probability as a reliability measure for their optimization.

Bashir and Sadeh [10] appreciated the importance of capacity sizing in order to fully meet demand due to uncertainty of wind and solar PV. As a measure of reliability they also used LPSP.

Zhang et al. [28] postulated that power supply reliability depended on optimum sizing of Solar PV, wind and Battery storage in a hybrid renewable energy supply system. They used LPSP as a reliability index and designed a system for a case study in Shengyang region.

Belmilli et al. [29] developed a software based on Loss of power supply probability algorithms for techno-economic analysis and optimization of hybrid systems. Other authors who considered LPSP in their work are quoted in references [30] and [4] LPSP has also been used alongside other indices in written literature. This usually done as means of ensuring that the proposed system other criteria other than reliability.

Bilal et al [6] desired to minimize the cost of their system whilst ensuring it was reliable enough. They used LPSP as reliability index alongside the annualized cost of the system which was to be maximized. Other authors aimed at reducing the cost of electricity generated by their system whilst meeting the required reliability index

Tafreshi et al. [7] , Paudel et al. [31] and Dial et al. [32] all used LPSP with levelized cost of electricity (LCE). Testa et al. [33] used LPSP alongside loss of power produced (LPP).

Another important index very widely used in power system reliability studies is the loss of load probability and the loss of load expectation. Using individual daily peak loads alongside a capacity outage probability table, the loss of load expectation can be defined as:

$$
\text { LOLE }=\sum_{i=1}^{n} P_{i}\left(C_{i}-L_{i}\right) \text { days } / \text { period }
$$

Where

$C_{i}$, is the available capacity on day $i$

$L_{i}$, is the forecast peak load on day $i$

$P_{i}$, is the probability of loss of load on day $i$, a value obtained directly from the capacity outage cumulative probability table.

The LOLE uses the daily peak load variation curve or the individual daily peak loads to calculate the expected number of days in the period considered that the peak load exceeds the available installed capacity. It is a measure of how long, on average, the available generation capacity is likely to fall short of the load demand. The LOLE is a sum of daily peak 
loss of load probabilities (LOLP) as it is an expectation.

The Loss of Load Probability (LOLP) is the projected amount of time in the long run that the load on a power system is expected to be greater than the capacity of the available generating capacity. It is formulated as

$$
\begin{gathered}
\text { LOLP }=\sum_{j} P\left[C_{i}=C_{j}\right] \cdot P\left[L>C_{j}\right] \\
=\sum_{j} P_{j} \cdot \frac{t_{j}}{100}
\end{gathered}
$$

Where

$P_{j}$ is the probability of capacity outage

$C_{j}$ is the remaining generation capacity

$t_{j}$ is the percentage of time when the load exceeds $C_{j}$

LOLP is related to the LOLE by

$$
L O L E=L O L P \times T
$$

Where $\mathrm{T}$ is the duration, given as 365 days for a model based on annual continuous load curve with day maximum loads or 8760 hours for an hourly load curve.

Ekren and Ekren [16] applied loss of load probability and autonomy analysis to determine the validity of their optimal sizing. In a later paper with a different optimization approach [15] they again used LLP to validate the reliability of their system.

\subsection{LOSS OF ENERGY INDICES}

The most popular loss of Energy Index encountered is the Expected Energy Not Served (EENS). When a loss of load expectation is calculated using a load duration curve, the area under the load duration curve represents the energy utilized in the specific period under consideration, an expected energy not supplied can be calculated. The result of this approach is usually represented as a probable ration between curtailed energy and energy needed by the system. This ratio, a very small figure, is termed the energy index of unreliability [27].

A more practical version is obtained by subtracting the energy index of unreliability from unity. The resultant index is known as the energy index of reliability.

Given,

$O_{k}$, Magnitude of the capacity outage.

$P_{k}$, Probability of a capacity outage equal to $O_{k}$

$E_{k}$, Energy curtailed by a capacity outage equal to $O_{k}$

Then the probable energy curtailed is presented is $E_{k} P_{k}$ and the loss of energy expectation is calculated as

$$
L O E E=\sum_{k=1}^{n} E_{k} P_{k}
$$

The normalized LOEE is obtained by dividing by the total energy under the duration curve, $E$

$$
L O E E_{p u}=\sum_{k=1}^{n} \frac{E_{k} P_{k}}{E}
$$

The per unit LOEE is mathematically equivalent to the energy index of unreliability, thus the EIR can be calculated as,

$$
E I R=1-L O E E_{p u}=1-\sum_{k=1}^{n} \frac{E_{k} P_{k}}{E}
$$

The Expected Energy Not Served is deduced as

$$
E E N S=C_{O i} \times P_{O i} \times T_{O i}(M W / \text { Year })
$$

Where

$C_{O i}$, is the capacity outage in $\mathrm{MW}$

$P_{O i}$, is the probability of capcity outage $i$

$T_{O i}$, is the time of capcity outage $i$ in (hours/year)

Arabali et al. [22] developed a stochastic framework for optimal sizing and reliability analysis of a hybrid power system including renewable energy source and energy storage. They used the Expected Energy Not Served index (EENS) with Energy Index of Reliability (EIR) to measure reliability of the system and a compromise solution method to arrive at the best compromise between reliability and cost.

Bahramirad and Reder [18] also used EENS as a probabilistic reliability calculation method to evaluate reliability of micro grids with energy storage systems in islanded operation.

\subsection{OTHER RELIABILITY INDICES}

Bashir and Sadeh [11] used the equivalent loss factor (ELF) as a reliability index to evaluate a hybrid system they had optimally sized. ELF was also used in [13]

Puri [34] considered the problem of sizing battery storage with renewable energy sources such as solar PV and wind using Fractional Load Not Served (FLNS) as a reliability criterion. He went ahead to demonstrate the characteristic differences between FLNS and LPSP which is the most common reliability index in written literature. He showed that for a fixed FLNS requirement, the minimum battery size required was a decreasing convex function of the size of the renewable energy source and that for a fixed size of renewable energy source, minimum battery size required was a decreasing convex function of FLNS. This is in contrast in his opinion to LPSP which does not display any convex relation to battery size.

Xydis [35] presented Exergetic Capacity Factor (ExCF) as a new parameter that can be used for better classification and evaluation of renewable energy sources. He examined both energy and exergy characteristics of wind and solar to determine factors that affect the exergy of a hybrid Wind Solar system

\subsection{ECONOMIC INDICES}

The most preferred indicator in economic modelling of the hybrid power system as observed from trends in recent literature is the levelized cost of electricity (LCE)[4].It is evaluated as 


$$
\mathrm{LCE}=\frac{\mathrm{TPV} \cdot \mathrm{CRF}}{E_{\text {load }}}
$$

Where

$T P V$ is the total present value of the system, given as a sum of present values of capital and maintenance for all the system components.

$$
T P V=C_{P V}+C_{W T G}+C_{b a t}
$$

$C R F$ is the capital recovery factor given as

$$
C R F=\frac{i(1+i)^{n}}{(1+i)^{n}-1}
$$

Where $i$ is the discount rate and $n$ the plant life.

Huang et al [14] used the levelized cost of electricity (LCOE) analysis method to compare the variation trends of power supplies which use different energy sources to generate.

Zhang et al. [2] used levelized cost of electricity (LCOE) with Deficiency of Power Supply Probability (DPSP) in the development of a methodology for calculation of the sizing and optimization of a stand-alone hybrid system.

\section{DISCUSSION}

Optimal sizing of hybrid renewable energy generation sources is a study area that has gained a lot of traction in recent times. From the literature reviewed clear distinctions can be seen in terms of methods used for optimization, generation technologies considered for hybridization, energy storage system employed, reliability index used to gauge the result of optimization, modelling methods used, simulation approaches and validation methods used.

The first clear distinction in the literature reviewed can be seen in the methods used for optimal sizing of capacities of key components of the hybrid renewable energy generation system. Search algorithms such as the dividing rectangles search algorithm are seen to be popular especially in circumstances where an optimal configuration is selected from a pool of possible configurations. Metaheuristic optimization algorithms, especially GA, PSO and their derivatives are seen to have significantly increased utilization from the reviewed works. This is a result of their relative maturity compared to other metaheuristics and their suitability to problems with large, non-smooth multimodal search spaces, the kind encountered in this research. Consequently for this same reason traditional gradient based approaches are not popular as they excel in smooth, small unimodal search spaces. Simulated Annealing, a heuristic method based on stochastic gradient search is also used in some works but doesn't seem to have the traction of GA and PSO in the field. A number of authors have also resorted to hybridized metaheuristics such as GA-PSO or PS-SMCS with GA initialization, these have had some success in reducing the time it takes to converge to an acceptable optimal solution. Some studies have also focused purely on optimization using available commercial software packages such as using Hybrid Optimization of Multiple Energy Resources (HOMER). Other software programs of similar nature that occur in literature as reviewed by Sunanda and Chandel[36] include HYBRID 2, RETScreen, iHOGA, INSEL, TRNSYS, iGRHYSO, HYBRIDS, RAPSIM, SOMES, SOLSTOR, HySim, HybSim, IPSYS, HySys, Dymola/Modelica, ARES, SOLSIM, and HYBRID DESIGNER. Moreover, a number of authors have used these commercial tools to validate their results. Other notable approaches for optimization encountered are Integer Programming methods, with both Mixed Nonlinear Integer Programing (MNIP) method and Mixed Linear Integer Programing (MLIP) method being encountered.

The second obvious distinction in the reviewed literature is on the technologies chosen for hybridization. The key principle behind the idea of hybridization is to take two intermittent source with complementary characteristics, optimally size them and use them in collaboration, this should consequently improve the overall system reliability. A key finding from the literature reviewed is that selection of technologies to be reviewed lies squarely on two factors: the available resources at the specific location considered and whether those resources have complementary regimes hence justify need for hybridization. From the literature reviewed the most popular resources considered for hybridization was wind with solar. This was implemented purely as wind and solar hybrid or in some cases with diesel generation, or with biofuel based generators, electrolizer with fuel cell and even tidal energy. Interestingly one author hybridized wind and pump storage hydro as well as conventional hydro power. The dominance of wind solar hybrids is a clearly attributed to their widespread availability when compared to other sources. Diesel generation added to the mix is usually to reduce system cost by reducing amount of capital spent on energy storage and in most cases the renewable components only serve to reduce fuel consumption. This approach is widely employed in industry today and in areas where diesel prices are fair. In this work the researcher's intention is to keep a pure renewable energy mix hence only wind and solar are considered.

The type or nature of energy storage system used is another line of possible classification of past works in this area. It was observed that energy storage was essential to converting renewable energy sources from their jerky intermittent state to a smoother and more reliable state by storing energy during peak production hours to provide ride through capability later on when renewable energy generation is insufficient to meet demand. Energy storage schemes from the literature reviewed have been seen to be application dependent in that, the suitability of a storage scheme depends solely on its intended application. Nonetheless it was observed that battery energy storage systems (BESS) were more versatile and found application in most situations in the literature reviewed. Other energy storage schemes encountered include: Hydrogen Storage both as gas and ingeniously in the form of the more stable 
methylcyclohexane as proposed in the Carbon Hydride Energy Storage System (CHES). Other popular storage schemes include Pumped Hydro Schemes which incidentally are the most efficient storage schemes but are only practical at very large scales, Flywheel Energy Storage Systems (FESS), Super Capacitors, and Compressed Air Energy Storage Systems (CAES). A noteworthy approach of hybridizing energy storage systems based on complementary characteristics was also encountered.

A performance index is necessary to evaluate the performance of the system in meeting its objectives. In most literature reviewed, there are two objectives along the lines of technical performance or reliability and economic performance. Consequently the indices used can be broadly and in very general terms classified into reliability performance indices and cost performance indices. Of the reliability indices used, the one with most traction is the loss of power supply probability. On the other hand, the common cost index in literature is the Levelized cost of electricity.

\section{CONCLUSION}

Even though other distinctions such as modelling method, simulation method and even validation method can be seen across the literature reviewed, it is clear that the two most important distinctions are in the optimization algorithms used and in the evaluation indices employed. Nonetheless, in a nutshell, the literature presented has given an overview on the trends, directions and possible inclination of research into optimal sizing of hybrid renewable energy systems. It has demonstrated that stand alone renewable energy generation is a viable alternative to grid supply or conventional fossil fuel basedpower generation for remote areas across the globe. Hybridizing two or more sources with complementary characteristics has emerged as an important technique for improving reliability and reducing cost of renewable energy generation in spite of the intermittency of the individual sources such as wind or solar. Additionally incorporation of a suitable energy storage solution has been shown to be key in converting the jerky intermittent energy sources to smoother, dispatchable forms. However the design, control and optimization of the hybrid power system is not a trivial task. Optimal sizing of the components of a hybrid system is crucial for the feasibility of such a system in terms of cost and reliability

\section{ACKNOWLEDGMENT}

This work has been successful due to the enabling environment provided by a number of factors. First and foremost, I would like to acknowledge the unwavering support and guidance of my co-author Prof. N. O. Abungu, the department of Electrical and Information engineering at the University of Nairobi, my colleagues at responsAbility Africa Ltd, friends and family. In particular I am grateful to the material and emotional support from my fiancée and my parents.

\section{REFERENCES}

[1]. R. Banos, F. Manzano-Agugliaro, F. G. Montoya, C.
Gil, A. Alcayde and J. Gomez, "Optimization methods applied to renewable and sustainable energy: A review," Renewable and Sustainable Energy Reviews, vol. 15, no. 4, pp. 1753-1766, 2011.

[2]. L. Zhang, R. Belfkira and G. Barakat, "Wind/PV/diesel energy system: Modeling and sizing optimization," Institute of Electrical and Electronics Engineers, September 1, 2011.

[3]. L. Zhang, G. Barakat and A. Yassine, "Design and optimal sizing of hybrid PV/wind/diesel system with battery storage by using DIRECT search algorithm," in Power Electronics and Motion Control Conference (EPE/PEMC), 2012 15th International, September 4, 2012.

[4]. R. Belfikra, O. Hajji, C. Nichita and G. Barakat, "Optimal sizing of stand-alone hybrid wind/PV system with battery storage," in Power Electronics and Applications, 2007 European Conference on, Aalborg, 2007.

[5]. H. Yang, W. Zhou, L. Lu and Z. Fang, "Optimal sizing method for stand-alone hybrid solar-wind system with LPSP technology by using genetic algorithm," Elsevier Journal of Solar Energy, vol. 82, pp. 354-367, 2008.

[6]. B. O. Bilal, V. Sambou, P. A. Ndiaye, C. M. Kebe and M. Ndongo, "Optimal design of a hybrid solarwind-battery system using the minimization of the annualized cost system and the minimization of the loss of power supply probability (LPSP)," Elsevier Journal of Renewable Energy, vol. 35, pp. 23882390, 2010.

[7]. M. S. Tafreshi, H. A. Zamani, S. M. Ezzati and M. Baghdadi, "Optimal unit sizing of Distributed Energy Resources in Micro Grid using genetic algorithm," in Electrical Engineering (ICEE), 2010 18th Iranian Conference on, Isfahan, Iran, 2010.

[8]. B. A. Jemaa, A. Hamzaoui, N. Essounbouli, F. Hnaien and F. Yalawi, "Optimum sizing of hybrid PV/wind/battery system using Fuzzy-Adaptive Genetic Algorithm," in Systems and Control (ICSC), 2013 3rd International Conference on, Algiers, 2013.

[9]. M. Pirhaghshenasvali and B. Asaei, "Optimal modeling and sizing of a practical hybrid wind/PV/diesel generation system," in Power Electronics, Drive Systems and Technologies Conference (PEDSTC), 2014 5th, Tehran, 2014.

[10]. M. Bashir and J. Sadeh, "Optimal sizing of hybrid wind/photovoltaic/battery considering the uncertainty of wind and photovoltaic power using Monte Carlo," in Environment and Electrical Engineering (EEEIC), 2012 11th International Conference on, Venice, 2012.

[11]. M. Bashir and J. Sadeh, "Size optimization of new hybrid stand-alone renewable energy system considering a reliability index," in Environment and Electrical Engineering (EEEIC), 2012 11th International Conference on, Venice, 2012.

[12]. Y. Saber and G. K. Venayagamoorthy, "Smart microgrid optimization with controllable loads using particle swarm optimization," in Power and Energy 
Society General Meeting (PES), 2013 IEEE, Vancouver, 2013.

[13]. Navaerfard, S. Tafreshi, M. Barzegari and A. Shahrood, "Optimal sizing of distributed energy resources in microgrid considering wind energy uncertainty with respect to reliability," in Energy Conference and Exhibition (EnergyCon), 2010 IEEE International, Manama, 2010.

[14]. J. He, C. Deng and W. Huang, "Optimal sizing of distributed generation in micro-grid considering Energy Price Equilibrium point analysis model," in Industrial Electronics and Applications (ICIEA), 2013 8th IEEE Conference on, Melbourne, 2013.

[15]. O. Ekren and B. Y. Ekren, "Size optimization of a $\mathrm{PV} /$ wind hybrid energy conversion system with battery storage using simulated annealing," Journal of Applied Energy, vol. 87, no. 2, pp. 592-598, 2010.

[16]. O. Ekren and B. Y. Ekren, "Size optimization of a $\mathrm{PV} /$ wind hybrid energy conversion system with battery storage using response surface methodology," Journal of Applied Energy, vol. 85, no. 11, pp. 1086 1101, 2008.

[17]. S. X. Chen and H. B. Gooi, "Sizing of energy storage system for microgrids," in Probabilistic Methods Applied to Power Systems (PMAPS), 2010 IEEE 11th International Conference on, Singapore, 2010.

[18]. S. Bahramirad and W. Reder, "Islanding applications of energy storage system," in Power and Energy Society General Meeting, 2012 IEEE, San Diego, 2012.

[19]. S. Chen, H. B. Gooi and M. Q. Wang, "Sizing of energy storage for microgrids," in Power and Energy Society General Meeting, 2012 IEEE, San Diego, 2012.

[20]. O. Ekren, B. Y. Ekren and O. Baris, "Break-even analysis and size optimization of a PV/wind hybrid energy conversion system with battery storage - A case study," Journal of Applied Energy, vol. 86, no. 7-8, pp. 1043-1054, 2009.

[21]. Crăciunescu, C. Popescu, M. Popescu and L. M. Florea, "Stand-alone hybrid wind-photovoltaic power generation systems optimal sizing," in $11 T H$ INTERNATIONAL CONFERENCE OF NUMERICAL ANALYSIS AND APPLIED MATHEMATICS 2013: ICNAAM 2013, Rhodes, 2013.

[22]. Arabali, M. Ghofrani, M. Etezadi-Amoli and M. S. Fadali, "Stochastic Performance Assessment and Sizing for a Hybrid Power System of Solar/Wind/Energy Storage," Sustainable Energy, IEEE Transactions on, vol. 5, no. 2, pp. 363-371, 2014.

[23]. P. C. Roy, A. Majumder and N. Chakraborty, "Optimization of a stand-alone Solar PV-Wind-DG Hybrid System for Distributed Power Generation at Sagar Island," in INTERNATIONAL CONFERENCE ON MODELING, OPTIMIZATION, AND COMPUTING (ICMOS 20110), West Bengal, 2010.

[24]. M. El Badawe, T. Iqbal and G. K. I. Mann, "Optimization and modeling of a stand-alone
wind/PV hybrid energy system," in Electrical \& Computer Engineering (CCECE), 2012 25th IEEE Canadian Conference on, Montreal, 2012.

[25]. S. Hearn, M. C. Lewis, S. B. Pratap, R. E. Hebner, F. M. Uriarte, R. G. Longoria and C. Donmei, "Utilization of Optimal Control Law to Size GridLevel Flywheel Energy Storage," Sustainable Energy, IEEE Transactions on, vol. 4, no. 3, pp. 611618, 2013.

[26]. J. S. Anagnostopoulos and D. E. Papantonis, "Simulation and size optimization of a pumpedstorage power plant for the recovery of wind-farms rejected energy," Journal of Renewable Energy, vol. 33, no. 7, pp. 1685-1694, 2008

[27]. Kamjoo, A. Maheri and G. Putrus, "Wind Speed and Solar Irradiance Variation Simulation Using ARMA Models in Design of Hybrid Wind-PV-Battery System," Journal of Clean Energy Technologies, vol. 1, no. 1, 2013.

[28]. F. Zhang, X. Chen, X. Yin and Z. Wang, "An improved capacity ratio design method based on complementary characteristics of wind and solar," in Electrical Machines and Systems (ICEMS), 2013 International Conference on, Busan, 2013.

[29]. H. Belmili, M. Haddadi, S. Bacha, . M. F. Almi and B. Bendib, "Sizing stand-alone photovoltaic-wind hybrid system: Techno-economic analysis and optimization," Renewable and Sustainable Energy Reviews, vol. 30, pp. 821 -832, 2013.

[30]. F. Z. Kadda, S. Zouggar and M. L. Elhafyani, "Optimal sizing of an autonomous hybrid system," in Renewable and Sustainable Energy Conference (IRSEC), 2013 International, Ouarzazate, 2013.

[31]. S. Paudel, J. N. Shrestha and J. A. Fereira, "Optimization of hybrid PV/wind power system for remote telecom station," in Power and Energy Systems (ICPS), 2011 International Conference on, Chennai, 2011.

[32]. S. Dial, D. Dial, M. Belhamel, M. Haddadi and A. Louche, "A methodology for optimal sizing of autonomous hybrid PV/wind system," Energy Policy, vol. 35, no. 11, pp. 5708-5718, 2007.

[33]. Testa, S. De Caro and T. Scimone, "Optimal structure selection for small-size hybrid renewable energy plants," in Power Electronics and Applications (EPE 2011), Proceedings of the 201114th European Conference on, Birmingham, 2011.

[34]. Puri, "Optimally sizing battery storage and renewable energy sources on an off-grid facility," in Power and Energy Society General Meeting (PES), 2013 IEEE, Vancouver, 2013.

[35]. G. Xydis, "On the exergetic capacity factor of a wind - solar power generation system," Journal of Cleaner Production, vol. 47, pp. 437-445, 2013.

[36]. S. Sinha and S. S. Chandel, "Review of Software Tools for Hybrid Renewable Energy," Renewable and Sustainable Energy Reviews, vol. 32, pp. 192205, 2014.

[37]. T. Skinner, H. T. Nguyen and D. K. Liu, "Performance Study of Multi-Deme Parallel Genetic 
Algorithm with Adaptive Mutation," in 2nd International Conference on Autonomous Robots and Agents, Palmerston North, New Zealand, 2004.

[38]. F. Scarlatache and G. Grigoras, "Optimal coordination of wind and hydro power plants in power systems," in Optimization of Electrical and Electronic Equipment (OPTIM), 2014 International Conference on, Bran, 2014.

[39]. J. Lorincz, T. Garma and G. Petrovic, "Measurements and Modelling of Base Station Power," Sensors, vol. 12, no. 1, pp. 4281-4310, 2012. Jemaa, A. Hamzaoui, N. Essounbouli, F. Hnaien and F. Yalawi, "Optimum sizing of hybrid PV/wind/battery system using Fuzzy-Adaptive Genetic Algorithm," in Systems and Control (ICSC), 2013 3rd International Conference on, Algiers, 2013.

[40]. Coley, An Introduction to Genetic Algorithms for Scientists and Engineers, London: World Scientific, 1999

[41]. National Renewable Energy Labaratory, "Statistical Wind Power Forecasting Models: Results forU.S. Wind Farms," in Windpower 2003, Austin, Texas, 2003.

\section{BIOGRAPHIES}

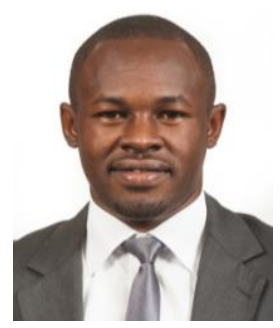

Victor O. Okinda was born in Kisumu, Kenya in 1988. He received his B.S. degree in electrical and electronics engineering from the University of Nairobi in 2013 and is currently pursuing an M.S. degree in electrical and electrical engineering from the University of Nairobi.

From 2014 he has worked as a project engineer for an investment firm in a research and project development capacity for renewable energy investments in East Africa.

His research interests include optimization, renewable energy generation technologies, hybrid power systems and energy storage.

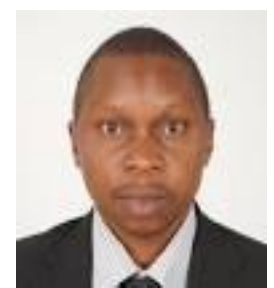

Nicodemus A. Odero received the B.S. and M.S. degrees in electrical and electronics engineering from the University of Nairobi - Kenya, in 1990,1993 and the Ph.D. degree in electrical engineering from the Jomo Kenyatta Uuniversity of Agriculture and Technology (JKUAT) in Nairboi in 2007.

He has worked in varous roles in research and teaching at both the university of Nairobi and JKUAT, becoming an associate professor in July 2014. He is also a registered practicing engineer and a member of IEEE. His research interests are in the area of power systems having published a number of papers in major journals and publications. 